University of Nebraska - Lincoln

DigitalCommons@University of Nebraska - Lincoln

Faculty Publications from the Department of Electrical \& Computer Engineering, Department Electrical and Computer Engineering

2011

\title{
Location Cheating: A Security Challenge to Location-based Social Network Services
}

\author{
Wenbo He \\ University of Nebraska-Lincoln, wenbohe@engr.unl.edu \\ Xue Liu \\ McGill University, xueliu@cs.mcgill.ca \\ Mai Ren \\ University of Nebraska-Lincoln, mren@cse.unl.edu
}

Follow this and additional works at: https://digitalcommons.unl.edu/electricalengineeringfacpub

Part of the Electrical and Computer Engineering Commons

He, Wenbo; Liu, Xue; and Ren, Mai, "Location Cheating: A Security Challenge to Location-based Social Network Services" (2011). Faculty Publications from the Department of Electrical and Computer Engineering. 205.

https://digitalcommons.unl.edu/electricalengineeringfacpub/205

This Article is brought to you for free and open access by the Electrical \& Computer Engineering, Department of at DigitalCommons@University of Nebraska - Lincoln. It has been accepted for inclusion in Faculty Publications from the Department of Electrical and Computer Engineering by an authorized administrator of DigitalCommons@University of Nebraska - Lincoln. 


\section{Location Cheating: A Security Challenge to Location-based Social Network Services}

\author{
Wenbo He \\ Electrical Engineering Department \\ University of Nebraska-Lincoln \\ Email: wenbohe@engr.unl.edu
}

\author{
Xue Liu \\ School of Computer Science \\ McGill University \\ Email: xueliu@cs.mcgill.ca
}

\author{
Mai Ren \\ Computer Science and Engineering \\ University of Nebraska-Lincoln \\ Email: mren@cse.unl.edu
}

\begin{abstract}
Location-based mobile social network services such as foursquare and Gowalla have grown exponentially over the past several years. These location-based services utilize the geographical position to enrich user experiences in a variety of contexts, including location-based searching and location-based mobile advertising. To attract more users, the location-based mobile social network services provide real-world rewards to the user, when a user checks in at a certain venue or location. This gives incentives for users to cheat on their locations. In this paper, we investigate the threat of location cheating attacks, find the root cause of the vulnerability, and outline the possible defending mechanisms. We use foursquare as an example to introduce a novel location cheating attack, which can easily pass the current location verification mechanism (e.g., cheater code of foursquare). We also crawl the foursquare website. By analyzing the crawled data, we show that automated large scale cheating is possible. Through this paper, we aim to call attention to location cheating in mobile social network services and provide insights into the defending mechanisms.
\end{abstract}

\section{INTRODUCTION}

A recent surge of location-based services (LBS) led by foursquare[1], Gowalla[2], GyPSii[3], Loopt[4], and Brightkite[5] has attracted a great deal of attention. Take foursquare as an example, it has become one of the top recommended applications for all smartphone platforms. Till August 2010, foursquare had attracted 1.89 million users since its launch in March 2009, and it draws in more than 10,000 new members daily. Meanwhile, hundreds of other similar services have been set up to follow this growing trend.

To encourage the use of location-based social network services, the service providers offer virtual or real-world rewards to a user if he or she checks in at a certain venue (i.e., places like coffee shops, restaurants, shopping malls). Foursquare provides real-world rewards (i.e., a free cup of coffee from Starbucks), which gives users incentives to cheat on their location information so that they can check in at a venue far away from where they really are.

1. We have obtained consent from foursquare to reveal the findings described in this paper.

2. The authors contributed equally to this work and are listed in alphabetical order.
In this paper, we use foursquare as an example to investigate the vulnerability in location-based social network services. The goal is to raise awareness of location cheating and suggest possible solutions to drive the success of the business model among service providers, registered venues, and users.

We first introduce a novel and practical attack on location cheating, where a user may claim he or she is at a certain location which is thousands of miles away from his/her actual location, thereby deceiving the service provider on location information. Though foursquare has adopted the cheater code to stop location cheating, we show that an attack can easily pass the cheater code. This benefits the attackers in the real world and can be more severe when combined with the analysis on venue (or location) profiles. In order to study foursquare's vulnerability to location cheating, we also crawled the foursquare website and used the crawling results to find suspicious cheaters on foursquare.

We found that the root cause of the vulnerability to location cheating is the lack of proper location verification mechanisms. If a user explores the open source operating systems for smart phones (e.g., Android) to modify global-positioning-system(GPS)-related application programming interfaces (APIs), the user is able to cheat on his/her location using falsified GPS information. Even if defending mechanisms like cheater code are deployed, the loosely regulated anticheating rules still leave space for location cheaters.

Our investigation suggests that defense against location cheating requires improvement to location verification ability. We outline the possible solutions to defense against location cheating. We suggest service providers take the following measures to prevent location cheating: (1) explore effective location verification technologies, and (2) limit profile crawling and analysis to mitigate the threat of location cheating. We believe that this investigation on location cheating will have a great impact on mobile social network services, and it will be an active research topic with strong practical value.

The rest of this paper is organized as follows. In Section II, we briefly describe the background of LBS and its associated business model, cheating scenarios and cheater code. In Section III, we introduce a basic location cheating attack, 
demonstrate how to automate the cheating, and optimize the benefits to the attacker through crawling and profile analysis. In Section IV, we show the results from our experiments on location cheating and examine the seriousness of current cheating threats. In Section V, we discuss possible solutions to prevent location cheating. In Section VI, we provide our conclusions and discuss future work.

\section{BACKGROUND}

In this section, we provide background and describe current practices used by location-based mobile social network services.

\section{A. Business Model}

Location-based social networking services allow users to share their location-related information. Users can add comments about a restaurant, find out what's happening, let their friends know where they are, and meet friends nearby for a cup of coffee. To report the geolocation to a service provider (e.g., foursquare[1]), a user needs to "check in" to the location/venue where the user is located. The service provider may broadcast the user's location information to his/her friends or even the public. The check-in is done by hand, which means a user is able to determine when he/she wants to check in, thereby controlling their location privacy. Services like this are not new, but they all have lacked incentives for people to use them, until foursquare introduced a new business model.

Foursquare uses a progressive reward mechanism to provide four types of reward incentives to its users. From the easiest to the hardest to get, they are: points, badges, mayorships, and real-world rewards. The first three are virtual rewards: (1) points are provided for all valid check-ins (e.g., the first time checking into a venue, checking into the same venue multiple times); (2) badges are awarded for specific achievements, such as "30 check-ins in a month" or "checked into 10 different venues"; and (3) mayorship of a venue is granted to the user who checked into that venue the most days in the past 60 days. Only the number of days with check-ins to this venue is counted, without consideration of how many checkins occurred per day or the total number of check-ins. Unlike points and badges which depend solely on a user's activities, the title of "Mayor" is given on a competition basis. There is only one mayor at each venue. This created a vulnerability in that if an attacker got the mayorship of this venue and kept checking into it every day, no other users could get the mayorship from the attacker.

The real-world rewards, like a free cup of coffee, were provided by businesses (e.g., restaurants or bars) who set up a partnership with foursquare. We crawled the information for all venues (discussed in more details later) and found that more than $90 \%$ percent of the rewards were only for mayors. This setup provides benefits to both foursquare and its partner businesses. On the other hand, the user's desire for discounts and the competition for the mayorship will likely bring more users (customers) to the partner businesses, increasing their profits. While the business model benefits multiple parties in the game, it makes foursquare a lucrative target to attack by location cheating.

\section{B. Possible Location Cheating Scenarios}

In the context of location-based social network services, a user may cheat on his/her location for various reasons. A user may want to get rewards from venues or impress others by claiming a false location. A business owner may use location cheating to check into a competing business, and badmouth that business by leaving bad comments.

Similar to most location-based social network services, foursquare initially relied on users' self-regulation to maintain the authenticity of the check-ins. Hence, the check-ins to any place a user can find in the foursquare client application (either using the suggested list of nearby venues, searching for a venue by name, or browsing and locating the venue on the map) were valid. Software is available on the market that can automatically check people into their desired venues, e.g., "Autosquare" for Android. The basic cheating method worked in the early days of foursquare. It is rather simple but obviously does not work now after the introduction of the location verification mechanism, which requires location information to complete the check-in process. However, the location cheaters can modify the location information and send a false location to the server.

The objective of the attacks is to automatically check into as many businesses as possible and as frequently as possible to maximize benefits through location cheating. A more sophisticated attack is automated cheating. To make automated cheating easier, the cheaters may use venue profile analysis to identify victims, which can be the venues who provide discounts or users who aim to get mayorship in specific venues. Hence, an attacker is able to select the venues where the "Mayor" title is less competitive and the rewards are more desirable or use a minimum number of check-ins to prevent another user from getting a mayorship.

\section{Cheater Code}

Foursquare has adopted the cheater code to defend against the location cheating attacks. One of its functions is to verify the location of a device by using the GPS function of that device. If a user claims that he/she is currently in a location far away from the location reported by the GPS of his/her phone, the check-in will be considered invalid and won't yield any rewards.

Apart from utilizing GPS for location verification, the cheater code also incorporates multiple rules which run on foursquare servers to determine if a user cheats on location. The details of the cheater code are concealed from users. But we managed to detect a few rules, through experiments, that are important to maneuvering location cheating to pass the scrutiny of the cheater code. A few of the criteria used in 
determining location cheating in the cheater code are listed as follows.

Frequent check-ins: We found a user cannot check into the same venue again within one hour. This rule prevents a user from checking in frequently to get as many points as possible and keep his/her name on top of the recent check-in list, making it more likely for people to contact the user for comments about the venue.

Super human speed: If a user continuously checks into locations that are located far away from each other, foursquare will indicate that the user is moving at "super human speed" and refuse to give any reward for his/her check-ins. This rule limits location cheating by a single user to a small geographic area.

Rapid-fire check-ins: If a user checks into multiple venues located within a 180 meter by 180 meter square area (which is well within a short walking distance such as in a mall) within a 1 minute interval, foursquare issues a warning about "rapidfire check-ins" on the fourth check-in. This rule stops a user from checking into multiple venues in a small area and within a short time period.

These rules essentially limit the number of check-ins a user can perform per day, thus reducing the potential for automated cheating. Clearly identifying these rules helps attackers to design the best way to work around them.

\section{LocAtion CheATing ATtACK}

In this section, we outline three levels of attack: cheating via GPS, automated cheating, and use of venue profile analysis to assist cheating. They severely interrupt the operation of LBSs when combined together. We first introduce four location cheating methods which can pass the validation from foursquare and other similar location-based social network services at least once. After that, we crawl data from foursquare's website and evade foursquare's cheater code to automate the cheating process. Finally, by analyzing the crawled data, we focus on a cheating attack on high-valued targets such as those who provide real-world rewards.

\section{A. Location Cheating Against GPS Verification}

Location-based services like foursquare use their client applications installed on their users' smartphones to get GPS location readings. Since this happens completely on the client side, it is relatively easy to hack. We analyzed foursquare's client application source code and confirmed that it gets the GPS location data from the phone's GPS-related APIs. Figure 1 shows the concept of such location cheating. Normally, the GPS module in a mobile phone will return the current location information to the LBS application, but an attacker blocks this and feeds fake location information to the LBS application so that it makes its server believe that this phone is really in the fake location. The cheating check-in will then be approved.

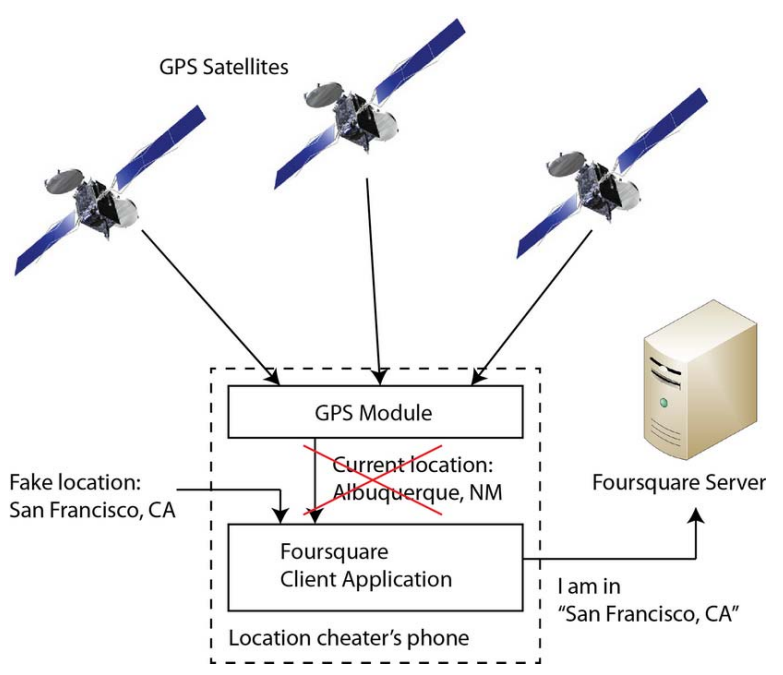

Fig. 1. Illustration of location cheating

There are several ways for an attacker to pass the GPS verification by providing foursquare's client application with fake GPS coordinates:

1) Via GPS APIs:

This method modifies the GPS-related APIs in a smartphone's operating system to let it return fake GPS data. This is easy because of the prevalence of open source smartphone operating systems like Android. These APIs can be modified to get GPS locations from sources other than the phone's GPS module, for example, from a server that returns fake GPS coordinates or simply from a local file. This method is limited to open source operating systems; but since LBSs like foursquare provide their client applications on major smartphone platforms (Android, iPhone, Blackberry), this is a universal cheating method. A hack into Android is representative to cover cross-platformed LBSs.

2) Via GPS module:

Directly hacking into a smartphone's GPS module is another way of cheating on location. There are two ways to do this: one via hardware and the other is via software. The former modifies the physical GPS hardware inside the phone, making it capable of faking data, so that the cheating is transparent to the mobile phone's operating system. The latter simulates a GPS device. For example, an attacker can write a program on a computer that simulates the behavior of a Bluetooth GPS receiver and let the phone connect to this simulated Bluetooth GPS receiver, enabling the simulated GPS to return fake coordinates. In fact, there are already a number of such tools on the market (e.g., Skylab GPS Simulator [6], Zyl Soft [7], GPS Generator Pro [8]), that were originally developed to help debug GPS-related software or gadgets. 
3) Via server provided APIs:

Foursquare provides a set of application APIs that allow developers to create new applications for them, like an application for uploading geotagged photos. These APIs can be employed by a location cheater to check into a place. The drawback is that not all LBS service providers provide such public server APIs. But this method is more convenient to issue a large-scale cheating attack.

4) Via device emulator:

Smartphone manufacturers (like Apple, Google, and Microsoft) provide device emulators to developers for easier debugging and testing. A device emulator is a full featured virtual machine of that device. One of the basic features of these device emulators is that they are configurable, including their simulated GPS module. Take the Android device emulator, for example. We can send it a specific command to set a location to the simulated GPS module. The GPS module of this emulator will return the coordinates we set to whichever application that needs GPS info. We conducted our experiments with this method, because this one is the easiest and most reliable when compared to the first two methods. Almost all potential attackers with a basic knowledge of mobile developing can master this method with no difficulty.

We chose an Android emulator to conduct our experiments. We've registered a user on foursquare for testing purposes and conducted all of our experiments in Albuquerque, New Mexico, and Lincoln, Nebraska. Our goal was to check into venues outside of the two states, so that we knew the cheating method was working. We used the tool "Dalvik Debug Monitor", which is part of Android SDK to connect to the emulator and set GPS coordinates in it. We found the coordinates of the target venues by looking up Google Earth, which shows the exact coordinates of where the mouse is pointing on its map.

The entire cheating process can be described as: hack the emulator; install and run foursquare application; find the coordinates of the target venue in Google Earth; use "Dalvik Debug Monitor" to set the coordinates in the emulator; find the target venue in the list of nearby venues in the foursquare application; and check into the target venue.

The results of our experiments showed that the check-ins to distant venues were all accepted, and we received rewards successfully. We got points for each of the check-ins, and we got badges like a normal user as well, i.e., after checking into ten different venues, we got the badge, "Adventurer: You've checked into ten different venues!". We also tried to get a mayorship, we chose the venue "Fisherman's Wharf Sign" in San Francisco, which is a well-known tourist spot, as the target venue; and we kept checking into it once a day for four consequence days. After nine days, we found our test user became the mayor of the venue. This experiment shows that the device emulator method works and can receive rewards.

\section{B. Crawling Data From Foursquare Website}

Getting the big picture of foursquare users and venues greatly helps in location cheating attacks, although the crawling itself is not an attack. There are two types of information that we crawled: users' profiles and venues' profiles. In this section, we describe the crawling procedure, in which we only accessed foursquare's public webpages. Wondracek, Holz, Kirda and Kruegel [9] introduced a similar crawling and attacking approach. We will also use the crawling results to show evidence of location cheating attacks on foursquare and identify the suspicious location cheaters in the next section.

To increase performance, we developed a multi-thread crawler to download and process a large amount of webpages (over 7 million). This architecture has been proven to be highly effective, for example, Cho, J. and Garcia-Molina used a parallel crawler to increase performance [10], and Chau, Pandit, Wang, Faloutsos focused on crawling social networks with parallel crawling [11].

We wrote the crawler in $\mathrm{C} \#$ and used MySQL as the database. We ran the crawler on three Windows PCs at the same time, each with a $2.0 \mathrm{GHz}$ Intel Core 2 Duo processor and 1GB RAM. The fourth computer with the same hardware specification, but running Ubuntu 8.10 server operating system and functions served as a database server. In our design, we set 14 to 16 threads on each of the three crawling machines to crawl 100,000 users per hour for user profile crawling, and set 5 to 6 threads on each machine to crawl around 50,000 venues per hour for venue profile crawling.

In total, we crawled more than 1.89 million users and 5.6 million venues, which agrees with foursquare's reported number of users. This means we can update all user profiles in less than two days or update all venue profiles in about five days. The crawling performance is an important design concern, because by repeatedly crawling data and comparing the differences between each set of crawling results, we can further investigate the behaviors of its users and extract more information. For example, the venue's recent visitor list does not have a time stamp to indicate when a user visited this venue; but if we crawl the venues daily, then we will be able to determine how frequently a user checks into a venue. We can further analyze the user behavior to show if the user is suspicious for location cheating.

Each user on foursquare has a profile that contains personal information. A user's profile provides information such as name, current location, check-in numbers, reward information, and list of friends. We cannot access the mayorships and check-in history directly (i.e., they are hidden from the public), since these two types of information may expose his/her location privacy. However, we can infer a user's mayorship information and partial check-in activities from venue profiles, which contain lists of recent visitors, and links to mayors. In addition, a venue profile also provides its name, address, location, number of users who checked in, unique visitors, and 
tips.

To crawl these profiles, we need to know the URLs of these profile pages. We discovered that foursquare uses continuously numbered IDs to identify their users and venues. By changing the ID in the URL, we can crawl almost all of the user and venue profiles. We believe this is a serious security weakness and should be patched soon.

Two types of URLs can be used to access user profiles. The first one is with an internal user ID in URL, like "http://foursquare.com/user/-1852791". To access another user with ID 23456, we just replace the "1852791" in the URL with "23456", and we can visit the public profile page with the new URL. We believe that all the users are accessible just by increasing or decreasing the user ID in the URL. We implemented a web crawler to do so, and we discovered around 1.89 million users in August 2010. Another type of URL contains the username, like "http://foursquare.com/user/test", where "test" is the username of a user. Not all users have a username-based URL as their profile page. Out of 1.89 million users, only $26.1 \%$ have a username, so we used the URL with ID in our crawling tool. For venue profiles, foursquare only uses numbered IDs in the URL of the profile pages, like "http://foursquare.com/venue/1235677".

After we had the URL of a profile page, we sent HTTP Get to this URL and got the HTML source code from the server's response. To extract data from the HTML source code, we let the crawler perform a set of regular expression matches. After extracting the data, we stored user and venue information in a database. Figure 2 shows the structure of the database; the arrows indicate the relationships between the tables. We stored user and venue profiles in tables UserInfo and VenueInfo respectively; and we also created a table called RecentCheckin to record the relations between venues and users. We put each venue's recent visitors in this table; and by counting the number of records for a user, we recorded the number of recent check-ins of this user and stored it in RecentCheckins of UserInfo. Similarly, by analyzing the MayorID of each venue, we calculated how many mayorships each user had and put the result in TotalMayors of UserInfo.

\section{Automated Cheating}

To achieve significant benefits from location cheating, attackers need to be able to control a large number of users and make them check in automatically. This requires the location cheaters to (1) find location coordinates of victim venues by computer program, and (2) automatically select a list of venues to check into pass the cheater code. We met the first requirement by crawling, and we could easily use SQL commands to get the location coordinates of the selected venues from the database.

Figure 3 shows the coordinates of all Starbucks branches in the US, where $x$ axes and $y$ axes are real coordinates. The location coordinates form the shape of United States territory,

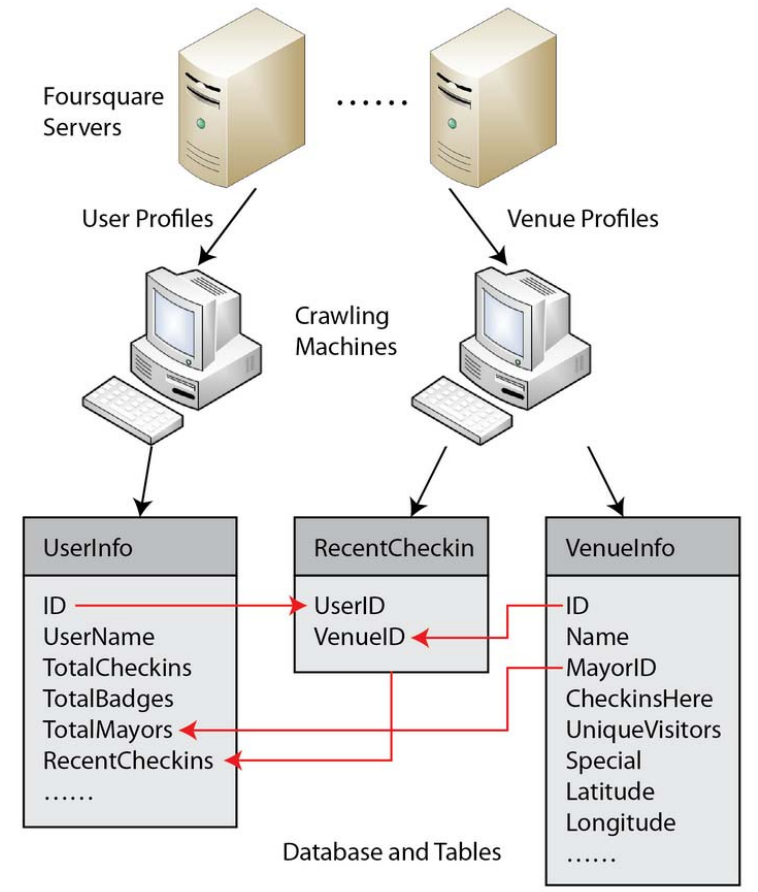

Fig. 2. A crawling architecture and the database to store crawled information from foursquare

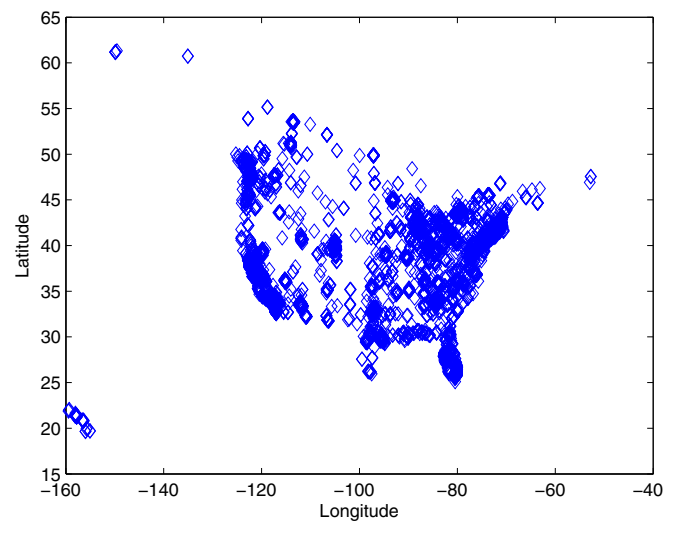

Fig. 3. Locations of Starbucks branches crawled from foursquare website

because Starbucks' branches are distributed all over the US. We draw this map by SQL command:

SELECT Longitude, Latitude FROM VenueInfo WHERE Name LIKE “\%Starbucks\%".

Second, to pass the anticheating verification, the key is to avoid triggering any of the rules in the cheater code, since it detects cheater on a per user basis, we focus on the strategy of a single user. An attacker needs to organize coordinates from the first step into a schedule, which states the sequence of venues to check into and the time interval a check-in has to wait after the previous check-in; and the schedule must follow 


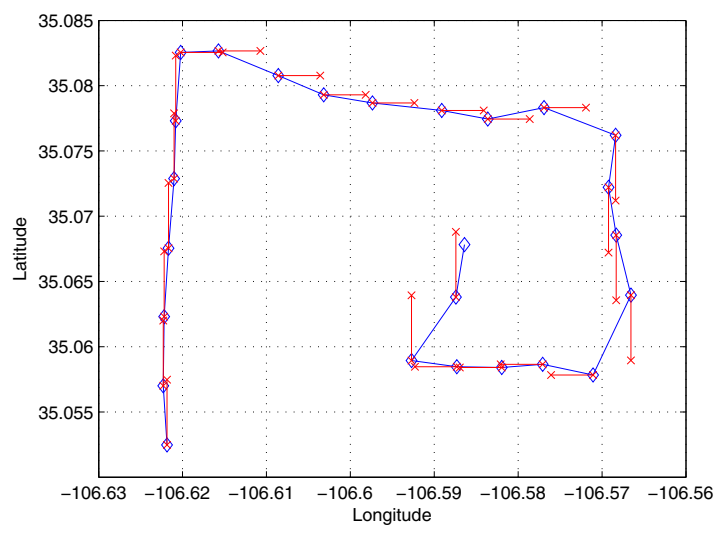

Fig. 4. An illustration of location cheating check-ins along a virtual path in the city.

all rules from the cheater code. The attacker could create a tool to do this automatically.

To determine the sequence of venues in which to check in, an attacker can create a virtual user, compute a virtual path to visit the target venues using Google Map's APIs, and build the check-in schedule along the virtual path. We also need to determine the time interval $T$ between check-ins, which is determined by distance between the check-ins in the schedule. Based on our experiments, we can check into venues less than 1 mile apart with a 5-minute interval without being detected as a cheater. So for distance $D$, less than 1 mile, we should set $T$ to 5 minutes; if $D>1$ mile, we let $T=D * 5$ minutes.

In our proof of concepts experiment, we created a semiautomatic location cheating tool. With the tool, an attacker can use any venue as the starting point. The attacker can then set the next cheating location by setting the moving direction and distance, for example, "move 500 yards to the west", the tool will search for the venue that is the closest to the target location and then automatically set the coordinate of the found venue to the emulator or generate a list for fully automated check-in later. The tool also automatically limits the process to avoid triggering any anticheating rules in the cheater code as we presented before.

Figure 4 shows the path of a virtual tour, the diamond points are the locations of venues the tool actually selected and checked into, and the cross-points and lines to them show the intended moving directions and target locations. The starting point in this tour is at the lower left point of Figure 4. We started by moving north and then kept turning right. The desired moving distance for each step was 0.005 degrees, either longitude or latitude, equivalent to about 550 meters in latitude direction or about 450 meters in longitude direction around this location. We set the interval between check-ins to 5 minutes since the moving distance is less than 1 mile. We continued checking into 25 venues without being detected as a cheater, and we received reward points and badges accordingly.

As we can see in Figure 4, for most of the time, the actual venues we checked into are not very far from the desired location, this is due to the high density of venues in the city. To move across large distances, we should increase the moving distance of each step, which will reduce the probability that we drift too much from the desired direction, like the second to last move in Figure 4.

\section{Cheating with Venue Profile Analysis}

Since the brute-force check-ins increase the chance that a cheater is caught, the location cheaters may gain intelligence from the venue analysis after the crawling. For example, an attacker may select the victim venues that provide special offers to their mayors and don't have a mayor yet (or are less competitive for mayorship) as targets. It is relatively easy to become the mayor of these venues. At the time this work is being prepared, around 1000 venues fall into this category.

Through profile analysis, we found a user in foursquare is the mayor of 865 venues but with a total check-in number of just 1265. It is interesting to observe that most of the 865 venues have no other visitors during the past 60 days, so only one check-in is enough to get the mayorship. We also discovered some special offers that do not require mayorship which are much easier to get, it's hard to find such information without crawling the venue profiles.

The attack can also target other users. For example, to stop a user from getting any mayorship, the attacker will analyze the venue profiles and find the venues that the victim user has been to or is the mayor. Then the attacker will apply an automated cheating attack on those venues in order to attack the mayorships of the victim.

\section{Evaluation of Location Cheating ON FOURSQUARE}

We have demonstrated that location cheating attack on foursquare. Next, we will show a big picture of location cheating through our crawling and analysis. In this section, we examine the signs of location cheating on foursquare. We found three identifying factors that related to location cheating. They are: (1) above normal level of activity, (2) below normal level of rewards, and (3) suspicious check-in patterns.

\section{A. High Check-in Frequency in Recent Visitor List}

If a user checks in too frequently and at too many venues, it is suspicious, because it is unlikely the user will visit too many places in a short amount of time.

We crawled the record of 20 million check-ins, and each of them represents a user visiting a venue once. That means, on average, each user on foursquare has checked into at least ten venues, and a venue has had at least four visitors. The actual number should be higher since only recent check-ins were shown on the website and were crawled. 


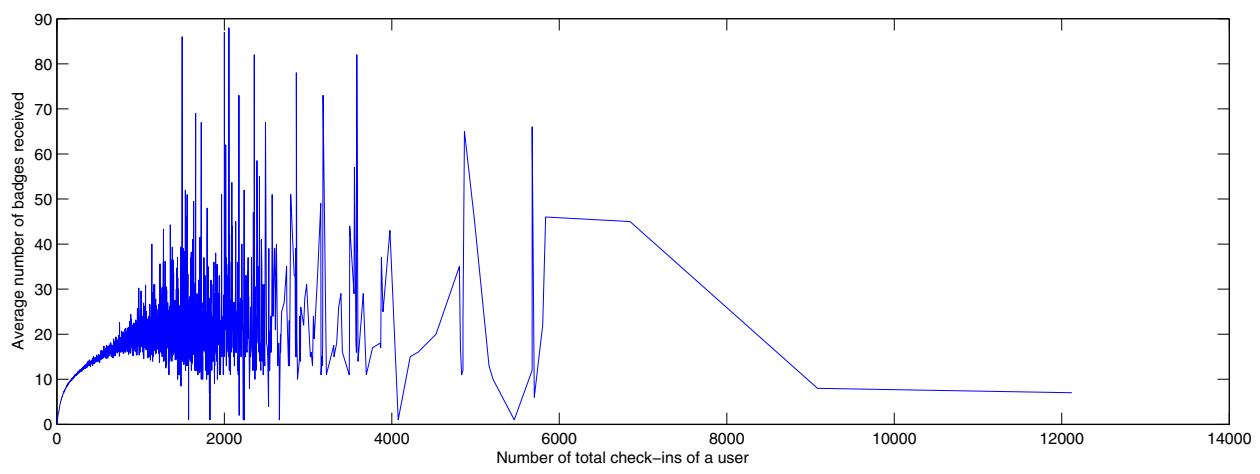

Fig. 5. Number of badges vs. number of check-ins: The average number of badges granted to users who have a certain number of total check-ins.

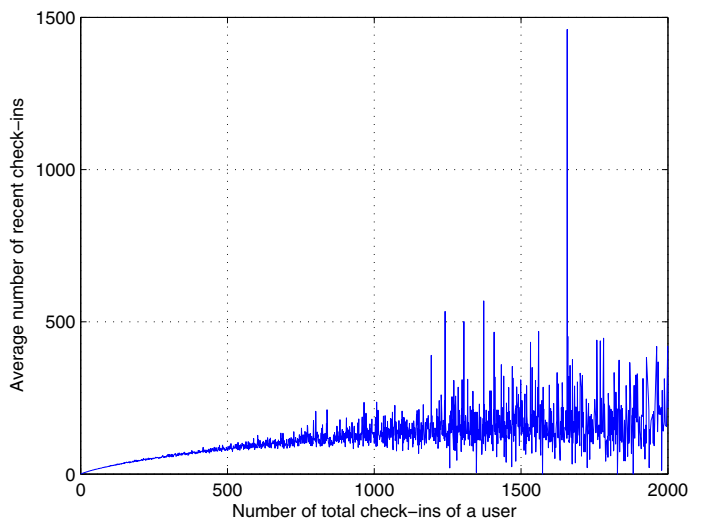

Fig. 6. Recent check-ins vs. total check-ins: the average recent check-ins of the users who have a certain number of total check-ins.

Figure 6 shows a relationship between the number of total check-ins and the recent check-ins. A recent check-in of a user means that the user is in a venue's recent visitor list, but we cannot directly know when this check-in happened. If this user is the only visitor of this venue, then he will be staying in the recent visitor list even if this check-in happened a year ago. In fact, there are 1,291,125 venues that have only one check-in; and 2,014,305 venues have had only one visitor ever. Though it is not a hard proof, the high ratio of recent check-ins to total check-ins of a user indicates that it is likely a user plays tricks to stay in the recent visits list, which is a sign of cheating. Here, we only included users with 2000 or less total check-ins since they cover $99.98 \%$ users. We get the number of recent check-ins vs. the number of total checkins of each user, and then we compute the average number of recent check-ins for users who have a given number of total check-ins (see Figure 6). We can see that some users with more than 1,000 check-ins have a unusually high percentage of recent check-ins, which suggests that those users are possibly cheaters, since it is not very likely for users to always check into a large number of different venues in a short time period.

From Figure 6, we can see that, on average, we get around 100 recent check-ins of a user, if the user did more than 500 check-ins total. There are 25,074 users that have a total checkin number falling in between 500 and 2000. It's not difficult to determine where they have been or are likely to go from this data.

\section{B. Low Reward Rate}

If a user has a large amount of check-ins but little rewards like badges, the user may have been detected as a cheater by foursquare so those check-ins were invalidated toward rewards, although they still increase the total check-in numbers of those users under foursquare's current policy. Figure 5 shows the relation between rewards (badges) and the number of checkins. We first get the number of badges vs. the number of total check-ins of each user, and then we compute the average number of badges for users who have a given number of total check-ins. As shown in Figure 5, for users with 1000 or less check-ins, the relation between the number of check-ins and badges is stable. It illustrates that a user will be likely to get more badges after doing more check-ins. It is reasonable because the rewards are usually granted to those who have checked in over a certain number of times to a venue. For the users with a larger number of check-ins, we can see that the curve in Figure 5 oscillates dramatically. Actually, many users with more than 1000 check-ins only have less than 10 badges. We think the best explanation for this is that they are location cheaters and got caught by foursquare and, thus, their check-ins yielded no rewards. For almost all users with more than 9000 check-ins, the reward level is low. The average check-ins per day for these users is over 16 times since the foursquare service was launched in March 2009, which is a strong evidence that these users are cheaters.

We notice that among 1.89 million users, 36.3\% have never checked into any venues, $20.4 \%$ have one to file check-ins, which means more than half of the users have only checked in less than six times. On the other hand, $0.2 \%$ of the users have 


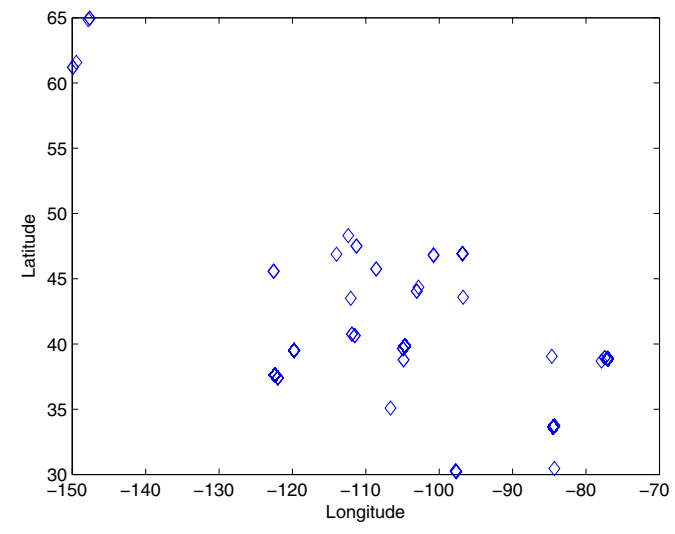

Fig. 7. Check-in locations of a suspected cheater

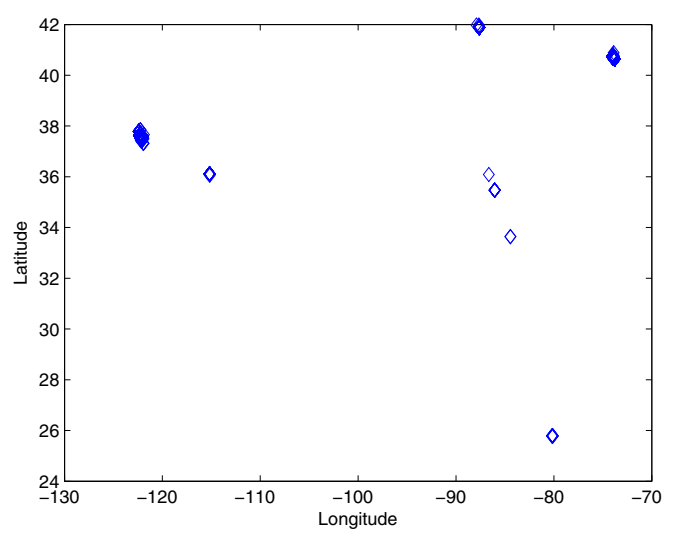

Fig. 8. Check-in locations of a "normal" user checked in at least 1,000 times; and 11 users have checked in at least 5,000 times. These 11 users who have made no less than 5,000 check-ins can be divided into two distinct groups by the number of mayorships they have. The first group has six users, each of whom is mayor of tens of venues, which are all concentrated in a city area. The other five users in the second group, including the one with over 12,000 check-ins, the highest among all users, do not have any mayorships, and they received much less badges than the first group. A further analysis indicates that four of the five users in the second group appeared in a recent visitor list of a venue, while the users in the first group are all in the recent visitor lists of a large amount of venues. This provides us with strong evidence that the users in the second group are cheaters and were caught, so their check-ins are invalidated.

\section{Suspicious Check-in Patterns}

Next, we will examine if the check-in pattern or history can tell if a user is a location cheater through further analysis of the crawled data.

We analyze a user's check-in pattern based on the recent check-in records. Figure 7 shows the recent check-in locations of a suspected cheater. We draw the venues to which a user has checked in on a map, so that we have a general idea of the places the user has "visited". This user is in the recent visitor lists of over 1000 venues. As we can see in Figure 7, those venues are scattered pretty far apart and spread over 30 different cities throughout the United States, including Alaska, and Europe. Judging from this user's ID (foursquare increments this ID as user registers), we believe that the user has used foursquare for less than one year. It illustrates that within a year, the user has "visited" at least 30 different cities, hence this user is suspected of location cheating.

Figure 8 shows the recent check-in locations of a user with a similar number of recent check-in records and similar ID (it means the two users registered for the foursquare service at almost the same time) as the user in Figure 7. But the venues he/she visited are concentrated in three cities (places with darker markers) and a few other places, where he may have visited on vacation. After examining the users with more than 1,000 recent check-in records, users with more than 2000 total check-ins, and users with more than 100 mayorships, we believe that the check-in pattern in Figure 8 is normal.

In the future, we will focus on those cheaters that haven't been detected by foursquare's current system. Foursquare implemented its cheater code anticheating system online around April 2010. Since then, all detected cheating check-ins are still count in the total number of check-ins, but do not receive any rewards. By the time this investigation was conducted (August, 2010), all mayors passed the scrutiny of the cheater code. So any cheaters we found in this group of users were new discoveries. There are 425,196 users who have the mayor title, and there are 2,315,747 venues which have mayors. On average, each user with a mayorship is the mayor of 5.45 venues. Those who are mayors of many venues are likely to be cheaters.

\section{Possible Solutions Against Location Cheating}

The investigation presented in this paper allows an attacker to launch automated location cheating attacks against a large number of victims, including service providers, business partners, and users. The root cause of the vulnerability is the lack of effective location verification mechanisms which can be deployed at a large scale. However, it is possible to counter these attacks. In this section, we list possible techniques to thwart the location cheating; and we suggest that location security be enhanced by limiting the access to user and venue profiles.

\section{A. Location Verification Techniques}

Distance bounding: Distance bounding protocols [12], [13], [14] that exploit the limitation on transmission range or speed of a communication signal for location verification, which does not rely on GPS inputs. This solution requires the deployment 
of verifiers around the registered venues, hence it will be expensive to deploy location verification based on distance bounding.

Address mapping: Using address mapping to geolocate IP addresses has been proposed in various applications, such as Tracert Map and Google Location Service. Researchers have adopted IP address mapping to locate mobile phones [15]. A challenge of applying IP address to verify location is that mobile phones may access the Internet from nonlocal IP addresses, and the IP addresses can be changed dynamically.

Venue side location verification: The $\mathrm{Wi}-\mathrm{Fi}$ routers that provide the Wi-Fi hotspot services can work as location verifiers. This technique provides an intrinsic distance bounding since only devices that are physically within the radio communication range of a $\mathrm{Wi}-\mathrm{Fi}$ router can communicate with it. According to previous literature [16], [17], the radio range of a Wi-Fi router is generally no more than one hundred meters. This range level enable use to identify cheaters that are miles away from the venue. However, for the cheaters within the transmission range a Wi-Fi router, this approach does not work. For example, a cheater sitting inside a McDonald's can check-in to the Wendy's next door, which is only 50 meters away. In this case, Wendy's owner can configure the $\mathrm{Wi}-\mathrm{Fi}$ router to limit the communicate within the restaurant via hardware or firmware configuration tools (i.e., DD-WRT [18]). In this solution, a Wi-Fi router takes the responsibility to measure if a check-in message was sent from a device in a legal area by checking the communication delay between the $\mathrm{Wi}-\mathrm{Fi}$ router and the device. If so, the Wi-Fi router sends the verification information to the corresponding LBS server.

In order to provide location verification service, the Wi-Fi routers must be registered to the LBS server and establish trusted communication with the server to block the impersonating attacks by location cheaters.

When comparing the three solutions, Distance Bounding provides the most accurate location data, and it can be used anywhere, but it is difficult to implement and has the highest cost. Address Mapping is the least accurate in terms of the location data it provides, it can be used anywhere, and it has the lowest cost and is the easiest to implement. Venue Side Location Verification has enough location accuracy, and it incurs no extra hardware purchase or installation cost for the venues. Owners of the venues can simply update the software on their existing routers to make these routers capable of defeating location cheaters.

\section{B. Mitigating Threat from Location Cheating}

As alluded above, with the assistance of profile analysis, an attacker may optimize the location cheating strategies. To limit the effect of potential location cheating attacks, we need to reduce the information exposed to the public. Along this direction we can employ the following techniques.
Access control for crawling: To prevent large-scale profile analysis by attackers, a direct solution is to take counter measures to stop or limit crawling. If a user must login to view the publicly available profile pages, it will be easier to detect the crawling users and block them. This can be combined with IP address blocking, if the service provider can detect the crawler's IP address. Even if the crawlers hide behind network address translations (NATs), blocking their IP addresses will cause limited collateral damage. Casado and Freedman [19] show most NATs only have a few hosts behind them, and proxies generally have much more. Crawling behind a public proxy cannot achieve enough performance. Although tools like Tor [20] may provide a high level of anonymity on the Internet, it also suffers from limited performance for crawling purpose.

Hiding information from profiles: To reduce the information leak, we hope that even if an attacker can successfully crawl the website, the information that can be extracted from the data is still limited. But if a subset of information in the profiles is removed, the usability of the location-based social networking service will be suffered. For example, if the recent check-in list is removed from the venue's profile, users cannot query the recent visitors to the venue for their comments about the venue. Hence, removing the information from profiles is not a good solution to prevent profile analysis. Rather, the service provider may use the hash function to hide necessary information (such as user IDs in the recent check-in list). Recently, the information leak has been studied. Griffith and Jakobsson [21] use public records to infer individuals' mothers' maiden names, and Heatherly et al. [22], as well as Zheleva and Getoor [23], show how public data provided by social networks can be used to infer private information.

\section{CONCLUSIONS AND FUTURE WORK}

In this paper, we introduced a novel and practical location cheating attack that enables an attacker to make the location-based service providers believe that the attacker is in a place far away from his/her real location. Through real world experiments on foursquare, the leading location-based social network, we demonstrate that our attacking approach works as expected; and location cheating really threatens the development and deployment of location-based mobile social network services. The counter measures against location cheating in current systems are not perfect.

We suggest several techniques for enhancing the security of location information. In the future, we will investigate to find better solutions to identify possible cheaters, especially those whom haven't been found by the existing anticheating mechanisms. We also would like to seek better solutions to the balance between the usability and the security in order to make the location-based mobile social networking service more attractive.

\section{REFERENCES}

[1] http://www. foursquare.com. 
[2] http://www.gowalla.com

[3] http://www.gypsii.com.

[4] http://www. loopt.com.

[5] http://www.brightkite.com

[6] http://www.skylab-mobilesystems.com/en/products / gps sim.html.

[7] http : //www. zylsoft.com/vgps.htm.

[8] http://www . avangardo. com/index.php?option= com content \&view=article\&id=47\&Itemid=55.

[9] G. Wondracek, T. Holz, E. Kirda, and C. Kruegel, "A practical attack to de-anonymize social network users," in 2010 IEEE Symposium on Security and Privacy. IEEE, 2010, pp. 223-238.

[10] J. Cho and H. Garcia-Molina, "Parallel crawlers," in Proceedings of the 11th international conference on World Wide Web. ACM, 2002, pp. 124-135.

[11] D. Chau, S. Pandit, S. Wang, and C. Faloutsos, "Parallel crawling for online social networks," in Proceedings of the 16th international conference on World Wide Web. ACM, 2007, pp. 1283-1284.

[12] G. Hancke and M. Kuhn, "An RFID distance bounding protocol," 2005.

[13] J. Chiang, J. Haas, and Y. Hu, "Secure and precise location verification using distance bounding and simultaneous multilateration," in Proceedings of the second ACM conference on Wireless network security. ACM, 2009, pp. 181-192.

[14] N. Sastry, U. Shankar, and D. Wagner, "Secure verification of location claims," in Proceedings of the 2nd ACM workshop on Wireless security. ACM, 2003, pp. 1-10.
[15] M. Balakrishnan, I. Mohomed, and V. Ramasubramanian, "Where's that phone?: geolocating IP addresses on 3G networks," in Proceedings of the 9th ACM SIGCOMM conference on Internet measurement conference. ACM, 2009, pp. 294-300.

[16] W. Lehr and L. McKnight, "Wireless Internet access: 3G vs. WiFi?* 1," Telecommunications Policy, vol. 27, no. 5-6, pp. 351-370, 2003.

[17] A. Howard, S. Siddiqi, and Sukhatme, "An experimental study of localization using wireless ethernet," in Field and Service Robotics. Springer, 2006, pp. 145-153.

[18] http: //www. dd-wrt. com.

[19] M. Casado and M. Freedman, "Peering through the shroud: The effect of edge opacity on IP-based client identification," in Proceedings of the 4th Networked Systems Design and Implementation, 2007.

[20] R. Dingledine, N. Mathewson, and P. Syverson, "Tor: The secondgeneration onion router," in Proceedings of the 13th conference on USENIX Security Symposium-Volume 13. USENIX Association, 2004, p. 21.

[21] V. Griffith and M. Jakobsson, "Messinwith Texas Deriving Mothers Maiden Names Using Public Records," in Applied Cryptography and Network Security. Springer, 2005, pp. 91-103.

[22] R. Heatherly, M. Kantarcioglu, B. Thuraisingham, and J. Lindamood, "Preventing private information inference attacks on social networks," 2009.

[23] E. Zheleva and L. Getoor, "To join or not to join: the illusion of privacy in social networks with mixed public and private user profiles," in Proceedings of the 18th international conference on World wide web. ACM, 2009, pp. 531-540. 